Este libro forma parte del acervo de la Biblioteca Jurídica Virtual del Instituto de Investigaciones Jurídicas de la UNAM

\title{
HABLEMOS DE LA MUERTE EN TIEMPOS DE PANDEMIA
}

\author{
Asunción ÁlVAREz DEL Río \\ Julieta GÓmez Ávalos
}

SumARIO: I. Introducción. II. Cuando los médicos no pueden curar. III. Decisiones sobre el final de la vida en la atención médica. IV. Asignación de recursos escasos en tiempos de pandemia. V. El manejo de los cuerpos fallecidos por COVID-19. VI. Los médicos y la muerte. VII. Conclusiones.

\section{INTRODUCGIÓN}

Formamos parte de una sociedad que igual que las demás sociedades occidentales tiene una gran dificultad para pensar o hablar de la muerte; ésta es una actitud que pretende negar la muerte y que fue desarrollándose respondiendo a varios factores relacionados entre sí. Mencionamos algunos: 1) el aumento de la esperanza de vida que fue llevando a ver la muerte como un acontecimiento lejano; 2) los avances de la ciencia y la medicina que influyeron en el aumento de años de vida y que no han dejado de avanzar (afortunadamente) haciendo posible curar enfermedades que antes significaban una sentencia de muerte y que permiten sustituir la función de órganos vitales prolongando así la vida de pacientes que antes hubieran fallecido; 3) la muerte que antes sucedía siempre en la casa se fue trasladando al hospital, con lo cual se fue perdiendo la experiencia familiar de acompañar y ver morir a los enfermos; los niños (y los no tan niños) dejaron de aprender de primera mano que la muerte es parte de la vida. Y debido a todos estos cambios que han favorecido el considerar a la muerte un asunto lejano, pareció menos necesario hablar de ella y dejamos de aprender a hacerlo, lo cual se vuelve un círculo vicioso: al surgir una situación relacionada con la muerte en la cual podría haber algo que decir, nos sentimos tan torpes que 
Este libro forma parte del acervo de la Biblioteca Jurídica Virtual del Instituto de Investigaciones Jurídicas de la UNAM

mejor guardamos silencio aumentando, claro, la torpeza y la necesidad de evitar la muerte.

El hecho de vivir en México introduce algunas particularidades. Por ejemplo, nos resulta muy fácil dar un lugar a la muerte en los días en que se celebra el Día de muertos con una fiesta llena de colorido y representaciones artísticas. De esta festividad se desprende un folklore que hace que nos resulten familiares algunas representaciones de la muerte (la Catrina) y algunas costumbres (comer pan de muertos o una calaverita de azúcar con nuestro nombre) que los extranjeros interpretan como si tuviéramos una relación muy amigable con la muerte. Ciertamente hay comunidades en nuestro país que conservan tradiciones que les permiten saber qué hacer cuando alguien va a morir y después de que falleció; saben acompañarse en el duelo a través de rituales que mitigan el dolor. Pero la mayoría de los mexicanos, fuera del 1 y 2 de noviembre, no habla de la muerte y cuando la enfrenta en una situación personal busca la manera de evadirla y hacer como que no está presente.

Como ha sucedido en el resto del mundo, la pandemia que ha cambiado nuestra vida nos ha confrontado con la realidad de la muerte y, junto con ella, con nuestra fragilidad. Las noticias y para muchos las experiencias de personas cercanas nos hacen ver que cualquiera puede contagiarse de COVID-19, tener la forma grave de la enfermedad y morir. Aunque sepamos que esto sólo sucede en un porcentaje bajo y que podemos hacer mucho para protegernos del virus, no tenemos la garantía de no enfermar. La confrontación más fuerte ha sido reconocer que no tenemos control sobre la muerte porque la medicina, por mucho que siga avanzando y por mucha fe que tengamos en ella, tiene límites para evitar que los pacientes mueran.

A continuación, las autoras vamos a hablar, manteniendo el formato de conversación que utilizamos en el coloquio virtual COVID-19 y bioética, de cómo vemos esta situación en los escenarios en que se está tratando a los enfermos graves por COVID-19. De cómo se toman las decisiones sobre los tratamientos; si los médicos, pacientes y familiares están preparados para enfrentar estas situaciones límites de la vida. ¿Cómo lo hacen? ¿Predomina la idea de que la muerte siempre se debe combatir? ¿Predomina el miedo de los pacientes y familiares que los lleva a pedir que se haga todo para curar al enfermo (con esta idea equivocada de que recibir tratamiento significa invariablemente curación)? ¿Se presentan pacientes con una voluntad anticipada en que manifiestan qué tratamientos no quieren recibir?

Relacionado con las preguntas anteriores, queremos hablar de un aspecto positivo de reconocer la realidad de la muerte porque nos permite influir y decidir sobre cómo morir y, antes de eso, cómo vivir y cómo no vivir 
Este libro forma parte del acervo de la Biblioteca Jurídica Virtual del Instituto de Investigaciones Jurídicas de la UNAM

el final de la vida. Si somos capaces de reflexionar sobre el hecho de que no tenemos el poder para evitar la muerte, podemos, como pacientes actuales o potenciales, como familiares o como médicos, voltear a ver eso sobre lo que sí tenemos control y que se expresa a través de las decisiones médicas sobre el final de la vida que la bioética ha debatido por décadas y sobre las que se han ido dando cambios legales en diferentes países. A estas decisiones hay que añadir los cuidados paliativos y los documentos de voluntad anticipada, todo lo cual sirve para influir en tener un mejor final de vida.

$\mathrm{Y}$ un último aspecto que nos ha parecido importante incluir en nuestra conversación es la relación del médico con la muerte; ahora no sólo de sus pacientes, sino de sus colegas y otros miembros de los equipos de salud, pues tristemente hemos visto que muchas de las personas que han estado atendiendo a pacientes o trabajando en los hospitales han perdido la vida. ¿Pueden prepararse los médicos a enfrentar estas pérdidas?, ¿`se les puede apoyar?

\section{GUANDO LOS MÉDICOS NO PUEDEN GURAR}

ASUNCIÓN: Julieta, además de tu formación de médica y tu amplia reflexión en el final de la vida desde la bioética, ahora que estás haciendo tu residencia en oncología y que se han tenido que hacer cambios en los hospitales para responder a la emergencia nacional que vivimos, has estado atendiendo pacientes de COVID-19, de manera que tienes una visión muy directa de lo que está pasando sobre el tema que nos ocupa. Me gustaría que nos pudieras platicar primero sobre los médicos, ¿cómo se están enfrentando en los hospitales las situaciones en que anticipan que los pacientes se van a morir, cuando se dan cuenta que la medicina no puede curarlos?, ¿deciden igualmente tratar de curar o hay casos en que, dadas las condiciones, saben que no es posible y solo dan cuidados paliativos?, ¿¿ no pueden saber realmente?

JULIETA: La realidad es que de por sí, en situaciones ordinarias, siempre es difícil saber hasta dónde hay que seguir tratando de curar. Hay tanto que se puede hacer gracias al desarrollo de la ciencia y la tecnología que es difícil establecer el límite y saber cuándo es el momento de detenerse. Por otro lado, los médicos están formados para actuar y no estamos acostumbrados a reflexionar y discutir sobre las situaciones que enfrentamos relacionadas con la muerte; son escasos los momentos en que se tienen este tipo de conversaciones. ${ }^{1}$

1 Álvarez, A. et al., "Experience of oncology residents with death: A qualitative study in Mexico", BMC Medical Ethics, 20:93, 2019, DOI: 10.1186/s12910-019-0432-4; Álvarez, A. et 
Este libro forma parte del acervo de la Biblioteca Jurídica Virtual del Instituto de Investigaciones Jurídicas de la UNAM

En una situación extraordinaria como la que vivimos, estamos aprendiendo en el día a día de la pandemia. Por eso es necesario que reflexionemos sobre lo que hay que hacer cuando atendemos a un paciente grave, intubado, por lo tanto, inconsciente por la sedación y que no cuenta con una voluntad anticipada. Sería importante poder hablar y discutir vía telefónica con su familiar sobre qué es lo que le hubiera gustado a su paciente en una situación de tal gravedad y con mal pronóstico. Pero no es tan sencillo; como el familiar no puede estar en el hospital, se quedó con la imagen con que dejó a su paciente cuando éste ingresó a urgencias y, junto con esa imagen, con sus enormes deseos de que los médicos pudieran curarlo. De ahí el reto de lograr que el familiar entienda y visualice cuál es la situación de gravedad de su paciente.

Desafortunadamente, por la falta de capacitación sobre la atención médica al final de la vida y también debido a la escasez de comités hospitalarios de bioética que asesoren a los médicos, es poco frecuente que en los hospitales se promueva la discusión sobre los dilemas éticos que enfrentamos y esto nos impide aprender de las situaciones que continuamente se presentan y que atendemos lo mejor que podemos, pero sabiendo que hay muchas cuestiones éticas que no estamos seguros de haber manejado de la mejor manera.

Por otro lado, es desalentador que en México no se le esté dando la importancia debida a los cuidados paliativos que deberían considerarse prioritarios siempre, pero especialmente en esta pandemia en que hay muchas situaciones en que sería lo único adecuado que ofrecer al paciente. Esto es consecuencia de que hay un desconocimiento de la medicina paliativa por parte de médicos, familiares y pacientes, además de una escasez de recursos humanos formados en cuidados paliativos.

Como conclusión a tu pregunta, diría que necesitamos estar más preparados para reconocer hasta dónde es conveniente tratar, identificar y valorar adecuadamente los datos de mal pronóstico que anticipan, con bastante certidumbre, una mala respuesta al tratamiento en pacientes de COVID-19. Y siempre que se pueda, es fundamental discutir la situación con el paciente o el familiar y que estos entiendan cuándo ya no se justifica dar tratamientos, pero que igualmente sepan que siempre se van a brindar cuidados paliativos oportunamente para que el enfermo tenga un buen final de vida.

ASUNCión: Mencionas varias cosas muy importantes y una que quisiera comentar es la importancia de comunicarse con los familiares. Como

al., "Facing Death in clinical practice: a view from physicians in Mexico", Archives of Medical Research 44(5), 2013, DOi: 10.1016/j.arcmed.2013.05.005. 
Este libro forma parte del acervo de la Biblioteca Jurídica Virtual del Instituto de Investigaciones Jurídicas de la UNAM

dices, se trata de una acción muy compleja y delicada, pero esencial. Aun con las limitaciones que señalas, se debe lograr una comunicación empática y veraz al mismo tiempo. Por otro lado, para el médico sería una gran ayuda saber si el paciente pensó antes de su internamiento sobre lo que querría, porque eso orientaría al médico sobre las decisiones que debe tomar. ¿Se ha podido hablar de eso?, ¿se puede preguntar a los pacientes?, ¿se hace?

Julieta: Totalmente de acuerdo. Son preguntas difíciles que no quisiéramos hacer ni responder, pero necesarias y que facilitan el actuar. Sí se puede y se debe preguntar. Me gustaría compartir el caso que tuve en una guardia COVID: ingresó un paciente de 30 años con obesidad e importante dificultad para respirar, pero sin verse afectada su saturación de oxígeno ni requerir un ventilador en ese momento. No tenía otras enfermedades, pero su estudio de imagen (tomografía) indicaba que tenía una afectación importante en los pulmones. Desde los primeros días decidimos preguntarle si aceptaría la intubación en caso de que llegar a deteriorarse respiratoriamente y la requiriera, explicándole todos los beneficios y riesgos. Él se sorprendió de que le hiciéramos esa pregunta; no se la esperaba, pero finalmente entendió la importancia de platicarlo y dijo que sí querría que lo intubaran. A los tres días de haber platicado con él, requirió intubación. Sin duda, es una pregunta fundamental que tenemos que hacer cuando recibimos a un paciente con COVID-19, porque puede darse un deterioro tan agudo y rápido en un paciente que después puede suceder que ya no haya la oportunidad de preguntarle y se tenga que decidir y actuar sin saber lo que el paciente realmente hubiera querido.

ASUNCión: Me imagino que debe suceder, quizá con frecuencia, que se pregunte al paciente o a los familiares (si el paciente ya no puede comunicarse) y digan que quieren que se haga todo. Ahí creo que será muy importante también la forma en que se comunique el médico y que no de falsas esperanzas

JULIETA: Claro, es vital una comunicación honesta y empática para que el paciente o el familiar, en caso de que el paciente no pueda comunicarse, pueda tener una decisión informada. El deber ético del médico es brindar información entendible e integral y aquí quiero citar al doctor Ruy Pérez Tamayo "El médico que no se involucra en su atención con el padecimiento integral del paciente, sino que se conforma con diagnosticar y tratar la enfermedad, o que lo abandona cuando ya ha agotado sus recursos terapéuticos curativos está cometiendo una grave falta de ética médica". ${ }^{2}$

2 Pérez Tamayo, R., "El medico y la muerte", Eutanasia. Hacia una muerte digna, México, Foro Consultivo Científico y Tecnológico, 2008, pp. 17-28. 
Este libro forma parte del acervo de la Biblioteca Jurídica Virtual del Instituto de Investigaciones Jurídicas de la UNAM

Por otro lado, es importante que los pacientes se responsabilicen de su enfermedad y muerte. Me ha tocado ver pacientes internados con múltiples comorbilidades y con mal pronóstico y aceptan ingresar a los hospitales con altas probabilidades de requerir intubación y padecer complicaciones, en lugar de elegir cómo morir. Desde luego es muy difícil esperar que actúen de otra forma si nunca han reflexionado en el final de su vida, en lo que podrían elegir. En la situación actual tan crítica es todavía más difícil tener con los pacientes las conversaciones necesarias que les ayuden a comprender y decidir lo mejor. Por eso, invito a los lectores a pensar, reflexionar y conversar con sus seres queridos sobre lo que querrían en caso de padecer una enfermedad muy grave en que no haya posibilidades de curarlos y que lo expresen por escrito a través de su voluntad anticipada.

Por cierto, ¿podrías comentar qué papel está teniendo la voluntad anticipada en la pandemia COVID-19?

ASUNCIÓN: Conviene aclarar que nos referimos al documento que una persona hace, cuando está competente, sobre los tratamientos que querría y no querría recibir en caso de encontrarse en una situación en que ya no pueda expresar su voluntad y otros deben tomar decisiones por ella. ${ }^{3}$ No se qué tanto se están presentando personas con su voluntad anticipada, pero sé, de manera anecdótica, que algunos notarios han dicho que en este periodo de pandemia los han buscado para hacer su voluntad anticipada más personas que en los más de diez años que van desde que se aprobó la ley en el DF. Eso significa que sí hay personas que han reflexionado sobre la posibilidad de enfermar y lo que querrían elegir, tal como ha sido reportado en el extranjero. ${ }^{4}$

\section{DEGISIONES SOBRE EL FINAL DE LA VIDA \\ EN LA ATENCIÓN MÉDICA}

ASUNCIÓN: En numerosas ocasiones hemos comentado tú y yo de lo importante que es que pacientes, familiares y médicos conozcan cuáles son las decisiones sobre el final de la vida que se permiten en México. Además, que comprendan que la razón por la que se ha reflexionado y discutido sobre

3 Álvarez, A. (coord.), La muerte asistida en México, Una opción más para morir con dignidad, Conceptos básicos sobre la atención médica al final de la vida, México, Por el Derecho a Morir con Dignidad, A. C, 2017, p. 119.

4 Auriemma, C. L. et al., "Completion of Advance Directives and Documented Care Preferences During the Coronavirus Disease 2019 (COVID-19) Pandemic", FAMA, 3, 2020, DOI:10.1001/jamanetworkopen.2020.15762. 
Este libro forma parte del acervo de la Biblioteca Jurídica Virtual del Instituto de Investigaciones Jurídicas de la UNAM

éstas, buscando acuerdos sobre lo que debe permitirse, responde al hecho de que en la atención médica inevitablemente llega el momento en que es necesario preguntarse si se deben seguir dando tratamientos para curar o prolongar la vida de un paciente o no. La pregunta es obligada porque no siempre representa un beneficio seguir dando estos tratamientos.

JUliETA: Sí, es muy importante que se conozcan estas decisiones. La primera es la limitación del esfuerzo terapéutico, cuando el médico propone detener o no iniciar un tratamiento, sea curativo o sea de soporte vital, porque ya no representa un beneficio para el paciente. La segunda es el rechazo al tratamiento y se refiere igualmente a detener o no iniciar acciones terapéuticas curativas o para prolongar la vida, pero aquí es el paciente quien no da su consentimiento a la propuesta del médico. Estas decisiones se consideran ética y legalmente aceptables, aun si como consecuencia de ellas el paciente muere.

A pesar de que en nuestro país se ha avanzado para que estas decisiones ayuden a que se dé una mejor atención en la última etapa de la vida y no se proporcionen tratamientos que sólo prolongan el final con mayor sufrimiento, en muchos hospitales, o no se conocen, o simplemente no se respetan.

Una decisión que hemos tenido que considerar en esta pandemia es la de la reanimación cardiopulmonar. En situaciones ordinarias se trata de discutir previamente con el paciente, dependiendo de sus enfermedades, el riesgo de un paro cardiaco. En caso de que éste ocurra y no se conozca la voluntad del paciente, la mayoría de las veces se activa el famoso "código azul" y el personal de salud acude inmediatamente para realizar la reanimación cardiopulmonar. En la situación extraordinaria que estamos viviendo con la COVID-19 todo ha cambiado: la evolución, ya que son pacientes que pueden tener un deterioro respiratorio en horas o minutos; la comunicación y la respuesta de los médicos. En una guardia nos tocó que personal de enfermería hablara vía telefónica a la sala de trabajo de médicos y avisara que un paciente no tenía signos, es decir se encontraba en paro cardiaco. Era un paciente de COVID-19 con múltiples comorbilidades y mal pronóstico que llevaba más de un mes intubado, no contaba con voluntad anticipada y sus familiares no habían comentado qué hacer en caso de un paro. El médico adscrito de la guardia comentó que enfermería iba a tomar el trazo electrocardiográfico para confirmar el paro y que no íbamos a vestirnos e ingresar ya que era fútil reanimarlo. Añadió que a la brevedad se iba a bajar el cuerpo a patología. Fue una experiencia muy diferente, ya que me sentí muy rara al no acudir inmediatamente a reanimar, de ahí mi interés en reflexionar y compartir la experiencia. 
Este libro forma parte del acervo de la Biblioteca Jurídica Virtual del Instituto de Investigaciones Jurídicas de la UNAM

Un punto importante que hay que señalar es que antes de acudir a una reanimación cardiopulmonar, se debe contar con todo el material de protección, ya que el riesgo de contagio durante esta maniobra es extremadamente alto, por el riesgo de generar aerosoles durante el procedimiento. La situación extraordinaria que ha impuesto la COVID-19 obliga a considerar si se justifica una intervención cuyo beneficio es cuestionable y que pondría en riesgo al personal de salud si no está debidamente protegido. Cuando no se cuenta con el material de protección necesario y se decide no reanimar es importante que el personal de salud sepa que no falta a su deber médico y que éticamente está justificado no intentar la reanimación. Desde luego, también deben saber de esta decisión con anticipación los pacientes y los familiares.

\section{ASIGNACIÓN DE REGURSOS ESGASOS EN TIEMPOS DE PANDEMIA}

JULIETA: Quizá podrías comentarnos sobre las diferencias que se dan al tomar decisiones sobre el final de la vida en las situaciones de escasez de recursos.

ASUNCiÓn: Para responder esto me voy a referir a la Guía Bioética para Asignación de Recursos Limitados de Medicina Crítica en Situación de Emergencia que publicó el Consejo de Salubridad General el 30 de abril y en cuya elaboración participamos varios miembros del Colegio de Bioética. ${ }^{5}$ Creo que las dos nos sentimos muy satisfechas de haber participado en ese interesante ejercicio de reflexión y discusión.

Ese documento se propuso para dar una respuesta a una situación que se estaba anticipando: que hubiera más pacientes que tratamientos disponibles. Se pensaba especialmente en la situación que estaba sucediendo en otros países en que había que tomar decisiones sobre a quién asignar ventiladores y camas en las unidades de cuidados intensivos. Era importante proponer un procedimiento que se sustentara desde la bioética y esto fue lo que ofreció la guía. A diferencia de lo que sucede en las situaciones ordinarias en que las decisiones sobre el final de la vida se orientan principalmente por los principios de beneficencia y respeto a la autonomía, en la situación de emergencia nacional que representa la pandemia, se debe poner por encima el principio de justicia buscando salvar el mayor número de vidas

5 Consejo de Salubridad General, Guía Bioética para Asignación de Recursos limitados de Medicina Crítica en Situación de Emergencia, disponible en: http://wrwre.csg.gob.mx/descargas/pdf/index/informacion_relevante/GuiaBioeticaTriaje_30_Abril_2020_7pm.pdf. 
Este libro forma parte del acervo de la Biblioteca Jurídica Virtual del Instituto de Investigaciones Jurídicas de la UNAM

posibles para utilizar los recursos escasos de la manera más ética. ${ }^{6}$ Mediante una valoración médica se evalúan las posibilidades que tiene cada paciente de aprovechar los tratamientos escasos y éstos se asignan a quienes pueden aprovecharlos. Vale la pena comentar que gracias a la importante discusión que despertó la publicación del borrador de la guía, se recibieron algunas críticas muy constructivas y como resultado de éstas, unido a la discusión sostenida con Conapred, se eliminaron todos los criterios que pudieran implicar algún tipo de discriminación. Es importante señalar que un objetivo muy importante de la guía es quitar a los médicos la tremenda responsabilidad de que sean ellos los que tomen las decisiones, con cada paciente, de a quién dar, y a quién no, un tratamiento que podría salvar su vida. Con la guía se busca librarlos de una responsabilidad que se ha visto que causa un enorme desgaste emocional en los médicos responsables de la atención de los pacientes. Por eso en la guía se establece que la decisión la tome un equipo de triage.

Otro punto que establece la guía es que a los pacientes que no reciban el tratamiento curativo (porque se anticipa que no podrán aprovecharlo) se les debe dar atención paliativa para que "tengan el mejor final de vida posible, de preferencia atendidos por personal de cuidados paliativos [...] Si la medicación no es suficiente para aliviar los síntomas se debe considerar la sedación, no con la intención de provocar la muerte, pero sí con la intención de que el paciente termine su vida sin sufrimiento". ${ }^{7}$ Así mismo, se recomienda que se busque la manera de que los pacientes y familiares puedan despedirse recurriendo, si es necesario, a la tecnología.

Julieta: ¿Y qué puedes decir sobre las decisiones sobre el final de la vida que aún nos falta tener a los mexicanos?

ASUNCiÓN: En México nos falta ampliar las opciones para elegir al final de la vida porque aún no es legal la muerte médicamente asistida que incluye la eutanasia y el suicidio médicamente asistido. Se trata de dos opciones de terminación de vida con las que una persona enferma, con un sufrimiento intolerable que no puede ser aliviado (ni con los cuidados paliativos) y que tiene la capacidad para tomar decisiones, elige adelantar su muerte para poner fin a ese sufrimiento. Pide la ayuda de un médico para tener una muerte segura y sin dolor.

6 Álvarez, A., Gómez, J., "Decisiones sobre el final de vida en una pandemia”, Nexos, mayo 2020, disponible en: https://wrere.nexos.com.mx/?p=47912.

7 Consejo de Salubridad General, op. cit. 
Este libro forma parte del acervo de la Biblioteca Jurídica Virtual del Instituto de Investigaciones Jurídicas de la UNAM

\section{EL MANEJO DE LOS GUERPOS FALLEGIDOS POR COVID-19}

ASUNCIÓN: Otro tema que es importante considerar es el que se refiere a los protocolos sobre el manejo del cuerpo de pacientes fallecidos por COVID-19, ¿nos puedes explicar cómo son?

Julieta: Para eso, me gustaría reconocer la participación de los trabajadores sociales que nos ha ayudado mucho a manejar esta parte de la atención. Son los que se encargan en los hospitales de estar en comunicación estrecha con los familiares de los pacientes COVID-19. Cuando existe una situación de gravedad, el equipo de trabajo social se los comunica y los guía sobre el proceso que debe seguirse en caso de fallecimiento. Como vivimos en una situación extraordinaria, es necesario explicar a la familia en términos sencillos que no deben tocar ni besar el cuerpo de su paciente por el riesgo de transmisión de la enfermedad. De hecho, existe una Guía para Manejo de Cadáveres por COVID-19 (SARS-CoV-2) en México, publicada en abril 2020 por la Secretaría de Salud de México. Aplaudo que se cuente con este tipo de recursos e invito a los lectores a revisarla. ${ }^{8}$

\section{LOS MÉDICOS Y LA MUERTE}

ASUnCIÓn: Como adelantábamos en la introducción, los médicos están teniendo una carga emocional muy fuerte porque no solo están enfrentando muchas muertes de pacientes, sino también de colegas. Además, viven con el miedo de contagiar a sus familiares. ¿Cómo has visto esto?, ¿qué has experimentado tú misma? Y, ¿cómo se puede ayudar a los médicos?, ¿qué se les puede recomendar?

JuliETA: Todo lo que comentas tiene un impacto muy importante en la salud física y mental de los médicos debido a que cotidianamente enfrentamos muertes de pacientes y, aunque con menos frecuencia, también de compañeros. De por sí somos un gremio que tiene una importante tasa de trastornos de ansiedad, de depresión y de suicidio y no estamos formados para pedir ayuda; más bien aprendemos a que tenemos que ser capaces de resolver todo por nosotros mismos. Entonces, ante estas experiencias tan difíciles, tendríamos, en primer lugar, que reconocer que necesitamos sentirnos acompañados

8 Secretaria de Salud. "Gobierno de México. Guía para Manejo de Cadáveres por COVID-19 (SARS-CoV-2) en México", México, abril de 2020, disponible en: https://coronavirus. gob.mx/wpcontent/uploads/2020/04/Guia_Manejo_Cadaveres_COVID-19.pdf. 
Este libro forma parte del acervo de la Biblioteca Jurídica Virtual del Instituto de Investigaciones Jurídicas de la UNAM

y estar dispuestos a pedir ayuda; tenemos que mejorar las redes de apoyo y acudir a terapias de soporte.

Cuando yo estaba por iniciar el trabajo en guardias COVID-19 me cambié de casa por el miedo a contagiar a mis papás. Por otro lado, he visto amigos, maestros y enfermeros infectarse; algunos se han recuperado, pero otros ya no están con nosotros. Afortunadamente, en el hospital donde laboro el equipo de salud mental da consulta virtualmente al personal que lo requiere. Esta pandemia ha mostrado uno de los puntos débiles en la formación de los médicos: no sabemos hablar de la muerte. Nunca vamos a ser lo suficientemente maduros para entender la muerte, pero si tuviéramos mejores herramientas durante nuestra formación, podríamos tener, con más facilidad, las conversaciones sobre ella que imponen las situaciones que enfrentamos. Esto permitiría tomar mejores decisiones junto con nuestros pacientes, lo cual nos haría sentir más seguros de darles una adecuada atención al final de su vida.

\section{CONCLUSIONES}

Después de este diálogo, podemos añadir que la muerte, aun si se da en un contexto de atención médica, no debe ser considerada sólo como un asunto médico; desde luego que es necesaria la atención del equipo de salud, pero no debe olvidarse que la muerte es, ante todo, un asunto humano y social; por ello, es importante preguntarse qué necesidades tiene un paciente que va a morir y cuál es la mejor forma de que muera. Se trata, también, de dejar de poner el acento en buscar cantidad de vida para voltear a privilegiar la calidad de vida.

En un artículo recientemente publicado en The Hastings Center Report que podría traducirse como Evitar el cuidado ineficaz al final de la vida: iuna lección del triaje?, Stephen Latham reflexiona sobre lo que podríamos estar aprendiendo de esta pandemia en que hemos tenido que encontrar el valor para enfrentar decisiones críticas. El tener que resolver cómo asignar recursos escasos que pueden salvar vidas nos ha permitido ver que en situaciones ordinarias muchas veces se toman malas decisiones porque se dan tratamientos a pacientes que no los van a aprovechar; son decisiones que se toman buscando sólo más cantidad de vida y, al mismo tiempo, que no reconocen los límites de la medicina ni las verdaderas obligaciones ante el enfermo. ${ }^{9}$

9 Latham, S. R., "Avoiding Ineffective End-of-Life Care: A Lesson from Triage?", The Hastings Center Report, 50, 2020, disponible en: https://doi.org/10.1002/hast.1141. 
Este libro forma parte del acervo de la Biblioteca Jurídica Virtual del Instituto de Investigaciones Jurídicas de la UNAM

Hemos comentado la importancia de que los médicos reflexionen sobre su propia muerte y sobre el crucial papel que juegan en el final de la vida de los pacientes que atienden, pero también creemos que ayudaría mucho que los pacientes y sus familiares, es decir todos, nos responsabilicemos de nuestra muerte, de cómo queremos y cómo no queremos que sea el final de nuestra vida para comunicarlo oportunamente y tomar mejores decisiones.

Finalmente, nos preguntamos cómo se responderá, después de la experiencia que vivimos en esta pandemia, la pregunta que se hacía Yuval Noah Harari hace dos meses en su artículo: " ${ }^{2} E l$ coronavirus cambiará nuestra actitud hacia la muerte?". ${ }^{10}$ Él anticipa que no, que en lugar de cambiar nuestra actitud de evasión y negación, la gente exigirá a los científicos que aceleren sus investigaciones para vencer a la muerte. Ojalá que Harari se equivoque y esta experiencia nos sirva para aprender a darle un lugar en nuestras reflexiones y conversaciones para estar mejor preparados para enfrentarla.

10 Harari, Y. N., “¿El coronavirus cambiará nuestra actitud hacia la muerte?”, Tribuna, abril de 2020, disponible en: https://blogs.elconfidencial.com/cultura/tribuna/2020-04-26/coronavirus-muerte-pandemia-harari_2564224/. 